(C) Masson, Paris, 1980.

Annales de Parasitologie (Paris)

1980 , t. $55, \mathrm{n}^{\circ} 6$, pp. 671-677.

\title{
La parasitose expérimentale à Scbistosoma mansoni du hamster doré
}

Étude de l'effet sur l'intensité du parasitisme et sur le taux d'anticorps circulants, après administration d'estradiol, de testostérone ou de progestérone

par A. BARRABES, T. H. DUONG, F. REYNOUARD et CH. COMBESCOT U.E.R. de Médecine et Pharmacie F 37000 Tours.

RESUME. Une diminution de l'intensité du parasitisme sous l'effet d'un implant de $20 \mathrm{mg}$ d'estradiol est à nouveau constatée mais un tel résultat est aussi obtenu avec la testostérone administrée de la même façon et aux mêmes doses. Par contre, aucune variation significative du nombre de parasites n'a été observée chez les animaux recevant un implant de $20 \mathrm{mg}$ de progestérone.

Par ailleurs, les examens sérologiques ont montré que les animaux traités par une de ces hormones ont tous un taux d'anticorps spécifiques plus élevé que les témoins non traités. Enfin, il n'y a aucune relation entre le taux d'anticorps sériques et le nombre de parasites hébergés.

Experimental Schistosoma mansoni parasitosis in golden hamsters. Effects on the intensity of parasitism, and on the rate of antibodies circulation, after administration of estrogene, testosterone, or progesterone.

SUMMARY. A reduction in the intensity of parasitism under the effect of an implantation of $20 \mathrm{mg}$ of estradiol is observed again but such result is also obtained with testostérone administered in the same way and at the same dose. On the other hand, no significant variation in the number of parasites was observed in animals hawing received an implantation of $20 \mathrm{mg}$ of progesterone.

Accepté le 30 juin 1980. 
However, serological examinations showed that the animals treated with one of these hormones all have a specific antibody level higher than the untreated control group. Finally, it is impossible to establish a relationship between level of serical antibodies and the number of parasites.

\section{Introduction}

Depuis 1970, nous poursuivons au Laboratoire de Parasitologie de Tours, des recherches ayant pour but de mettre en évidence une influence éventuelle de l'état hormonal de l'hôte, sur l'aptitude de ce dernier à contracter une parasitose. C'est ainsi qu'il a été établi qu'une importante imprégnation estrogénique diminuait, chez les hamsters femelles, le nombre de schistosomes adultes hébergés après infestation expérimentale par des furcocercaires de Schistosoma mansoni (4-5-6). Parallèlement l'un d'entre nous a constaté que, dans la parasitose expérimentale du hamster à Dipetalonema viteae, les femelles sont moins parasitées que les mâles (9). Ces diverses observations nous ont amenés à rechercher si d'autres hormones sexuelles ayant une structure voisine de celle de l'estradiol, n'avaient pas une action similaire ; la progestérone et la testostérone ont été ainsi expérimentées.

De plus, au cours de ce travail, nous avons effectué des dosages d'anticorps spécifiques afin de préciser s'il existait une corrélation entre le taux d'anticorps sériques et l'intensité du parasitisme.

La présente communication rend compte de nos résultats.

\section{Protocole expérimental}

Quarante hamsters femelles, d'un poids moyen de $90 \mathrm{~g}$, sont castrés par voie latérodorsale, après anesthésie au pentobarbital sodique, puis répartis en 4 groupes de 10 animaux. Quatorze jours après la castration, les hamsters reçoivent un implant soit de $20 \mathrm{mg}$ de progestérone (Groupe I), soit de $20 \mathrm{mg}$ de testostérone (Groupe II), soit de $20 \mathrm{mg}$ d'estradiol (Groupe III) ; les animaux du groupe IV qui ne reçoivent aucun traitement hormonal servent de témoins. Quatorze jours après la mise en place des implants, chaque animal est parasité par une injection intra-péritonéale d'une suspension de 100 furcocercaires de Schistosoma mansoni (souche antillaise). Trente six jours après l'infestation, les hamsters sont sacrifiés afin d'apprécier l'intensité de la parasitose. Le comptage des vers s'effectue au niveau du foie, par perfusion, puis écrasement de l'organe entre 2 plaques de verre, mais aussi dans les vaisseaux mésentériques. Tous les détails techniques de ce protocole ont été développés par ailleurs (1). Lors du sacrifice nous recueillons le sang de chaque animal sur tube sec et séparons les sérums afin de réaliser un dosage des anticorps spécifiques circulants. Nous utilisons pour celà la réaction d'immunofluorescence indirecte sur coupe de schistosomes adultes (7). 


\section{Résultats}

Les résultats concernant l'intensité de la parasitose sont consignés dans le tableau I qui fait apparaître le nombre de vers trouvés chez chaque nimal, la moyenne pour chacun des 4 groupes et la variance. On voit que le nombre moyen de schistosomes observés chez les hamsters témoins est, de 22 ; il est de 12 pour les hamsters traités à l'estradiol, de 25 pour ceux qui ont reçu un implant de progestérone et de 13 pour les animaux traités à la testostérone. La comparaison des variances de chacun des groupes I,

Tableau I. Effet de l'cestradiol, de la progestérone et de la testostérone sur l'intensité de la parasitose expérimentale à Schistosoma Mansoni du hamster doré femelle castré.

\begin{tabular}{|c|c|c|c|c|c|c|c|c|}
\hline & 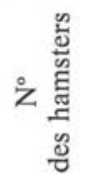 & 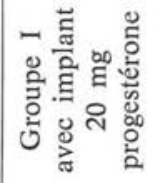 & 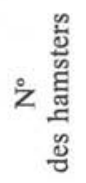 & 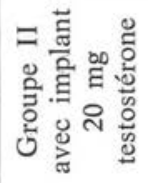 & 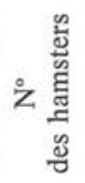 & 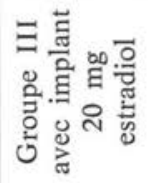 & 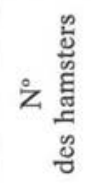 & 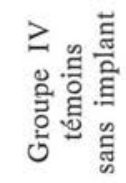 \\
\hline 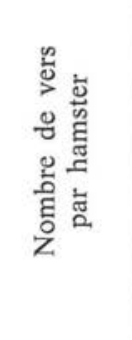 & $\begin{array}{l}11 \\
12 \\
13 \\
14 \\
15 \\
16 \\
17 \\
18 \\
19 \\
20\end{array}$ & $\begin{array}{l}20 \\
14 \\
22 \\
42 \\
18 \\
14 \\
32 \\
44 \\
32 \\
18\end{array}$ & $\begin{array}{l}21 \\
22 \\
23 \\
24 \\
25 \\
26 \\
27 \\
28 \\
29 \\
30\end{array}$ & $\begin{array}{r}8 \\
8 \\
10 \\
10 \\
28 \\
20 \\
18 \\
10 \\
/ \\
10\end{array}$ & $\begin{array}{r}1 \\
2 \\
3 \\
4 \\
5 \\
6 \\
7 \\
8 \\
9 \\
10\end{array}$ & $\begin{array}{r}4 \\
8 \\
22 \\
12 \\
22 \\
4 \\
22 \\
6 \\
/ \\
12\end{array}$ & $\begin{array}{l}31 \\
32 \\
33 \\
34 \\
35 \\
36 \\
37 \\
38 \\
39 \\
40\end{array}$ & $\begin{array}{c}34 \\
14 \\
10 \\
12 \\
26 \\
24 \\
32 \\
22 \\
/ \\
/\end{array}$ \\
\hline Moyenne & & 25 & & 13 & & 12 & & 22 \\
\hline $\begin{array}{c}\text { Variance } \\
\mathrm{S}^{2}\end{array}$ & & 124,3 & & 47,7 & & 59,7 & & 81,7 \\
\hline
\end{tabular}

II et III avec la variance obtenue pour le groupe témoin IV nous montre que pour chaque comparaison, les 2 variances ne diffèrent jamais significativement au risque de $5 \%$. La loi de probabilité étant normale nous avons comparé les nombres moyens de vers obtenus dans les 4 lots à l'aide du rapport $\mathrm{F}$. Ce qui nous a permis de conclure que les moyennes diffèrent significativement dans leur ensemble au risque de $1 \%$ $(\mathrm{F}=4,76$ avec les degrés de liberté 3 et 32$)$. Nous avons ensuite à l'aide du test $t$ (Student) comparé le nombre moyen de vers trouvés chez les animaux traités respectivement par la progestérone, la testostérone ou l'estradiol au nombre moyen de vers hébergés par les témoins (tableau II). Ce résultat confirme à nouveau l'action protectrice d'un implant de $20 \mathrm{mg}$ d'estradiol; la différence des nombres moyens de vers 
entre le groupe III, ayant reçu de l'estradiol et le groupe IV témoin étant significative à mieux que $5 \%$. Avec la testostérone, une diminution du nombre moyen de vers est également notée ; elle est du même ordre que celle observée avec l'estradiol et l'étude statistique des résultats nous permet de la considérer comme significative. Par contre, l'administration de progestérone ne diminue pas d'une façon significative l'intensité de la parasitose.

Tableau II. Comparaison des nombres moyens de vers par Hamster dans les groupes I, II et III recevant respectivement $20 \mathrm{mg}$ de progestérone, de testostérone ou d'estradiol au nombre moyen de vers par hamster chez les témoins du groupe IV ne recevant pas d'hormone.

\begin{tabular}{c|c|c|c}
\hline & $\begin{array}{c}\text { Groupe I } \\
\text { Hamsters \& castrés } \\
\text { avec implant de } \\
20 \mathrm{mg} \text { de } \\
\text { progestérone }\end{array}$ & $\begin{array}{c}\text { Groupe II } \\
\text { Hamsters \& castrés } \\
\text { avec implant de } \\
20 \mathrm{mg} \mathrm{de} \\
\text { testostérone }\end{array}$ & $\begin{array}{c}\text { Groupe III } \\
\text { Hamsters o castrés } \\
\text { avec implant de } \\
20 \mathrm{mg} \text { d'estradiol }\end{array}$ \\
\hline $\begin{array}{c}\text { Valeur } t \\
\begin{array}{c}\text { Degré de liberté } \\
\text { D.D.L. }\end{array}\end{array}$ & 0,79 & 2,11 & 2,29 \\
\hline & 16 & 15 & 15
\end{tabular}

Sur le tableau III sont portés les taux de positivité des différents sérums ainsi que les valeurs obtenues après transformation logarithmique du réciproque de chaque dilution. Pour chacun des groupes nous avons pu ainsi calculer la Geometrical Mean Reciprocal Titer (G.M.R.T.) *. Pour le groupe I ayant reçu $20 \mathrm{mg}$ de progestérone, la G.M.R.T. est 79,43 , elle est de 35,48 pour les animaux du groupe II traités à la testostérone, de 70,79 pour le groupe III recevant l'estradiol et de 43,35 pour les hamsters témoins du groupe IV. Nous avons comparé à l'aide du test U de Mann-Whitney les taux de positivité obtenus chez les différents lots de hamsters traités aux hormones aux taux de positivité notés chez les animaux témoins. Nous avons pour les lots I (traité à la progestérone) et III (traité à l'estradiol) un taux d'anticorps significativement plus élevé que chez les témoins. Entre le groupe II traité à la testostérone, et le groupe témoins, la différence n'est pas significative.

Par ailleurs, afin de rechercher une éventuelle corrélation entre le nombre de parasites hébergés par chaque animal en expérience au moment du sacrifice et le taux d'anticorps circulants spécifiques dans son sérum au même moment nous avons pour chacun des groupes calculé le coefficient de corrélation après transformation logarithmique des taux de dilution. Les valeurs des coefficients de corrélation calculées ont permis de conclure à l'indépendance des deux variables.

(*) GMRT (Geometrical Mean Reciprocal Titer) se calcule selon la formule antilog $\Sigma \frac{f(\log x)}{\mathrm{N}}$ où $x$ représente l'inverse des différents titres d'AC, $f$ le nombre de sérums pour chacun des titres et $n$ le nombre total de sérums. 


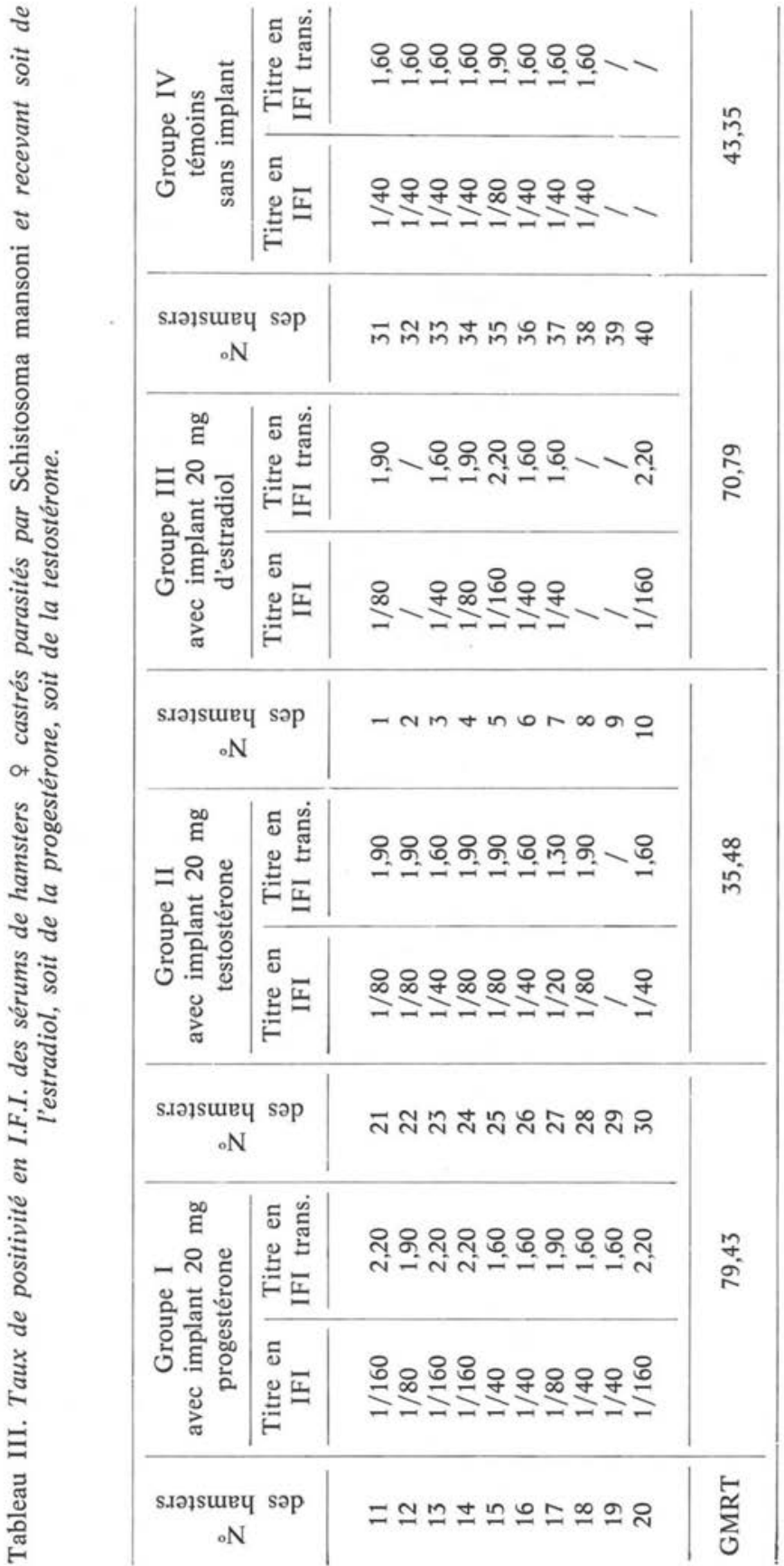




\section{Discussion}

La diminution de l'intensité du parasitisme à Schistosoma mansoni observée chez les hamsters sous l'effet de l'administration d'implants d'estradiol à $20 \mathrm{mg}$ ne doit pas avoir pour seule cause la structure stéroïdique de la molécule du produit administré, sinon une action similaire devrait être observée avec d'autres substances de structure chimique voisine telles la progestérone et la testostérone. Or, nos résultats montrent que si la testostérone agit bien dans le même sens que l'estradiol, la progestérone par contre entraîne, par rapport aux témoins, une légère augmentation du nombre moyen de vers hébergés ; cependant, la différence observée étant très faible et non significative, on peut conclure que la progestérone n'a pas eu, dans nos conditions expérimentales, d'influence sur l'intensité du parasitisme des hamsters. Il convient cependant de remarquer que les implants de progestérone utilisés étaient dosés à $20 \mathrm{mg}$ comme ceux de testostérone et d'estradiol; or, en thérapeutique les doses du progestérone administrées sont toujours supérieures aux doses d'estradiol et de testostérone. $\mathrm{Si}$ la progestérone n'a pas modifié de façon significative l'intensité du parasitisme chez ces animaux la raison en est peut-être que le dosage en hormones des comprimés pour implantation utilisés, était trop faible. Cette dernière hypothèse est d'ailleurs en accord avec d'autres travaux faits par l'un d'entre nous et non publiés qui montrent que l'effet protecteur de l'estradiol ne se manifeste qu'avec des doses d'hormones importantes.

Par ailleurs, on peut remarquer que pour la testostérone nos résultats sont en accord avec ceux obtenus par Berg (1) qui a expérimenté sur la parasitose à Schistosoma mansoni de la souris mâle castrée ; cet auteur a également noté une diminution sous l'influence d'injections de testostérone, du nombre de vers hébergés par animal. Bussolati et coll. (2) ont aussi signalé chez la souris mâle parasitée par Schistosoma mansoni une augmentation de la résistance à la parasitose sous l'influence d'injections de méthyl testostérone. Nous citerons aussi les travaux de Lagrange (7) qui signale une prolongation de la survie de souris mâles parasitées par Schistosoma mansoni sous l'influence combinée de l'estradiol, de la progestérone et des ultrasons.

\section{Conclusion}

L'administration à des hamsters femelles castrés, d'implants de testostérone renfermant $20 \mathrm{mg}$ d'hormone diminue d'une façon significative l'intensité de la parasitose à Schistosoma mansoni obtenue en injectant par voie intra-péritonéale des furcocercaires de cette espèce. Cette action est comparable à celle qui est obtenue avec des implants de $20 \mathrm{mg}$ d'estradiol. Par contre, la progestérone administrée de la même façon et aux mêmes doses que la testostérone et l'estradiol, n'a pas eu d'influence sur le nombre de vers hébergés par les hamsters dans les conditions de l'expérience. 
Par ailleurs, des examens sérologiques effectués par immunofluorescence, au moment du sacrifice, soit 36 jours après l'infestation, montrent tout d'abord que les animaux recevant soit de l'estradiol, soit de la progestérone, ont un G.M.R.T. significativement plus élevé que les témoins. Les animaux recevant de la testostérone eux ont un GMRT inférieur mais la différence observée n'est pas significative.

Enfin il n'y a aucune corrélation entre le nombre de vers hébergés par les hamsters dans les différents groupes et le taux d'anticorps circulants (doses par IFI) présents dans leur sérum. Ceci au $36^{\mathrm{e}}$ jour de la parasitose.

\section{Bibliographie}

1. Barrabes A. : Action des hormones sexuelles au cours de la parasitose expérimentale à Schistosoma mansoni du hamster doré femelle. Thèse pour le Doctorat de Pharmacie, Diplôme d'Université, 1974.

2. Berg E.: Effects of castration and testostérone in male mice on Schistosoma mansoni. Trans. R. Soc. Trop. Med. Hyg., 1957, 51, 353-358.

3. Bussolati C., de Carneri I., Castellino S., Varinoni V., Sperzani G. L.: Traitement of experimental and clinical schistosomiasis with hormonal inhibitors of ovulation. Am. J. Trop. Med. Hyg., 1957, 6, 497-499.

4. Combescot Ch., Barrabes A., Reynouard F., Demaret J.: Effet protecteur des hormones sexuelles dans la parasitose expérimentale à Schistosoma mansoni du hamster doré femelle. $1^{\text {er }}$ Multicolloque Européen de Parasitologie, Rennes (France), 1971.

5. Combescot Ch., Barrabes A., Reynouard F.: Effet protecteur de l'estradiol au cours de la parasitose expérimentale à Schistosoma mansoni chez le hamster doré femelle castré, importance de la barrière cutanée. Ann. Parasitol., Hum Comp., 1974, 49, 185-189.

6. Combescot Ch., Barrabes A., Gerhardt R.: L'estradiol ne modifie pas la proportion des vers mâles et femelles dans la bilharziose expérimentale à Schistosoma mansoni du hamster doré femelle. Ann. Parasitol. Hum. Comp., 1975, 50, 629-633.

7. Coudert J., Garin J.-P., Ambroise-Thomas P., Pothier M.-A., Kien Truong Thai : Immunofluorescence sur coupe à la congélation de Schistosoma mansoni. Acta Trop., 1968, 25, 109-132.

8. Lagrange E.: Action des ultrasons sur la bilharziose expérimentale de la souris. C.R. Soc. Biol., Paris, 1962, 1725-1727.

9. Reynouard F., Barrabes A., Combescot Ch.: Influence du sexe de l'hôte définitif de la filariose expérimentale à Dipetelonema vitae du hamster doré Cricetus auratus, présentée à la Société française de Parasitologie, Paris. 